\title{
La toma de decisiones en la política española de transporte: aportación y limitaciones de la evaluación de proyectos
}

\author{
Ángel Aparicio \\ Universidad Politécnica de Madrid
}

\section{Resumen}

Se analiza en este trabajo el proceso de toma de decisiones y las herramientas de evaluación de proyectos empleadas por el Ministerio de Fomento, con el fin de identificar qué elementos pueden haber consolidado y estar justificando hoy la continuidad de una política fuertemente inversora, con escasa rentabilidad socioeconómica y poco coherente con una perspectiva de desarrollo sostenible. La conclusión es que la influencia real de las herramientas de análisis coste beneficio (ACB) es modesta, y que la búsqueda de políticas más coherentes exigirían, más que una mayor influencia de dichas herramientas, una profunda revisión del sistema de toma de decisiones, incluyendo la incorporación de los instrumentos ligados a la evaluación estratégica en las fases iniciales de planificación, un mayor acceso de la sociedad a la información sobre los proyectos objeto de evaluación y un esfuerzo de transparencia y apertura en los procesos de diálogo y concertación. Se plantean algunas propuestas de mejora del conjunto del sistema de evaluación y toma de decisiones, incluyendo el papel que el $A C B$ podría tener en ese nuevo marco.

Palabras clave: evaluación, transporte, gobernanza, planificación, análisis coste-beneficio, evaluación ambiental estratégica.

Clasificación JEL: D61, H43, L92, L93, Q51.

\begin{abstract}
This paper analyses the decision-making process at the Spanish Ministry of Transport, and the assessment tools used to support it. Its objective is to identify which elements within the decisionmaking system are helping to justify the implementation of costly projects with low socio-economic benefits and doubtful consistence with the objectives of sustainable transport policies. The conclusion is that the actual influence of cost/benefit assessment (CBA) tools on decision making is weak, and that the development of more consistent policies cannot rely on CBA tools, but would need an in-depth reform of the current decision-making framework, including the empowerment of the Strategic Environmental Assessment process at the earliest planning stages, improved access of the public to information on transport plans and projects, and considerable effort at improving transparency and opening-up participation within the currently closed institutional consensus-building dialogue. The paper makes a number of proposals to improve the assessment and decision-making process, including some thoughts on the role that CBA could play in this new framework.

Keywords: assessment, transport, governance, planning, cost-benefit, strategic environmental assesment.

JEL Classification: D61, H43, L92, L93, Q51.
\end{abstract}




\section{Introducción}

Con una inversión en infraestructuras de transporte que desde el Ministerio de Fomento se ha mantenido por encima del 1,3 por 100 del PIB prácticamente durante toda una década, España ha sido probablemente el país europeo con una mayor inversión relativa en grandes infraestructuras de transporte. Esta política inversora se inicia a mediados de los años 80, facilitada por una generosa aportación de fondos de la Unión Europea. Con el paso del tiempo y la puesta en marcha de nuevos proyectos, ha ido creciendo la preocupación sobre la rentabilidad socioeconómica de algunas de las inversiones seleccionadas y, en definitiva, por la racionalidad de su ejecución, pero este cuestionamiento, focalizado en el ámbito académico, rara vez ha llegado al debate político o mediático, a pesar de resultar un hecho sobradamente conocido: en 2004, el propio Plan Estratégico de Infraestructuras y Transporte constataba, en su diagnóstico, la existencia de un porcentaje considerable de la red de autopistas y autovías con tráfico débil.

En este artículo se trata de identificar algunas de las causas que posibilitan la continuidad de una política decididamente inversora en infraestructuras de transporte, y que puede caracterizarse como absolutamente singular en el ámbito de los países desarrollados y con niveles de dotación similares -si no algo inferiores-a los de España. Para ello se analiza tanto el proceso de toma de decisiones como las herramientas de evaluación de proyectos empleadas por el Ministerio de Fomento. La conclusión es que no parece que la selección de proyectos con escasa rentabilidad pueda ser el resultado de un empleo deficiente de las herramientas disponibles para la evaluación socioeconómica de proyectos. Un mayor peso de los resultados de estas herramientas en la toma de decisiones resultaría probablemente en una política continuista en términos de inversión, si bien con una distribución territorial y modal diferente. Por el contrario, la búsqueda de políticas más coherentes con los objetivos generalmente admitidos del desarrollo sostenible exigirían una profunda revisión del propio sistema de toma de decisiones, especialmente en los momentos iniciales de la planificación, incluyendo la incorporación de los instrumentos ligados a la evaluación estratégica en esas fases, el acceso a la información sobre los proyectos objeto de evaluación o la transparencia y apertura en los procesos de diálogo y concertación.

El artículo se estructura en cuatro apartados: en el primero, se establece el marco conceptual en el que puede situarse teóricamente la evaluación de proyectos dentro de un proceso de toma de decisiones; en el segundo, se describe el proceso de toma de decisiones actual en el Ministerio de Fomento, y algunos antecedentes que clarifican el porqué se ha llegado a este tipo de configuración; en el tercero, se analizan las principales debilidades de este sistema de toma de decisiones, tanto desde una perspectiva de eficiencia como de buena gobernanza; en el cuarto apartado se analiza cuál puede ser la contribución de los instrumentos de evaluación económica más allá de su papel tradicional de comparación entre variantes de un mismo proyecto. Finalmente, se plantean algunas propuestas de mejora del conjunto del sistema de 
evaluación y toma de decisiones, incluyendo el papel que el análisis coste-beneficio (ACB) podría tener en ese nuevo marco.

\section{Evaluación de proyectos y toma de decisiones: marco conceptual}

Para el análisis del proceso de toma de decisiones de una política pública, es conveniente distinguir dos aspectos complementarios: en primer lugar, cómo llegan a consolidarse, a constituirse, unas determinadas prácticas a través de una evolución más o menos compleja; en segundo lugar, en qué medida esas prácticas consolidadas de facto se encuentran formalizadas, es decir, siguen procedimientos claramente explicitados y mayoritariamente aceptados. El análisis en estas dos vertientes del proceso nos llevará a considerar las aportaciones que se realizan en cada momento desde el ámbito técnico y político, a sus interacciones y, en definitiva a cuál es la delimitación entre ambos campos.

Desde la perspectiva de la constitución de las prácticas hoy al uso en España, es de notar que la evaluación técnica ha evolucionado de manera considerable en las últimas décadas, tanto en su contenido como en su relación con la toma de decisiones. La evaluación técnica toma inicialmente como variable fundamental la demanda de transporte, con una perspectiva de ingeniería de transporte, y progresivamente acomete la cuantificación en términos monetarios de esa demanda y de sus efectos. La lógica escasez de recursos lleva a buscar una mayor integración entre estas consideraciones puramente técnicas y los costes del proyecto: esa integración se consigue con el Análisis Coste-Beneficio (ACB), bien consolidado ya en los años 60. A esta evaluación se une, muy posteriormente, la evaluación del impacto ambiental como consecuencia de la integración en las Comunidades Europeas y de la obligación de aplicar la Directiva 85/337/CE sobre evaluación del impacto ambiental de proyectos. La aplicación de la Directiva $85 / 337 / \mathrm{CE}^{1}$ va a tener una fuerte influencia sobre el conjunto del sistema de evaluación y su relación con la toma de decisiones, al contribuir decisivamente a formalizar un sistema de toma de decisiones sobre el proyecto basado en la utilización de sistemas de análisis multicriterio (AMC), en los que se integra la evaluación económica, en la evaluación ambiental y en los resultados de la información pública realizada a partir de la información anterior.

Progresivamente va tomando cuerpo una evaluación complementaria, menos estructurada, ligada a los efectos territoriales (también llamados a veces efectos indirectos) de la actuación. Si bien algunos de estos efectos se incluyen en ocasiones dentro del ACB o figuran dentro del AMC, se carece de una sistemática de análisis y, más frecuentemente, se producen estudios ad hoc para analizarlos. Proliferan así estudios dispares, sin una metodología consolidada, planteados generalmente desde una perspectiva un tanto apriorística sobre la importancia y bondad de ese tipo de efectos. Además, la carencia, en general, de un marco formalizado de ordenación

\footnotetext{
${ }^{1}$ Incorporada al ordenamiento jurídico nacional mediante el Real Decreto Legislativo 1302/1986.
} 
del territorio dificulta cualquier intento de análisis sistematizado de estos efectos $\mathrm{y}$, en definitiva, de establecer una metodología técnica rigurosa.

Por su parte, la evolución del proceso de toma de decisiones viene marcada por consideraciones no sólo de eficiencia, sino de legitimidad. La búsqueda de legitimidad no puede basarse únicamente en la eficiencia de la decisión, es decir, en la racionalidad económica, la disponibilidad de recursos financieros o la rapidez en la tramitación y ejecución del proyecto. La legitimidad busca ampliar en lo posible el apoyo a la toma de decisión, orientada, en principio, a la ejecución de la actuación ${ }^{2}$, mediante la suma de agentes significativos a través de procesos que pueden variar desde la simple información hasta el diálogo, el debate o la negociación.

En cuanto a la formalización, la evaluación de actuaciones y la toma de decisiones también han seguido procesos diferentes, si bien con relaciones entre ellos. En la medida en que dicha formalización se produce, los dos procesos ganan en transparencia, y resulta más sencillo proceder a su revisión y a su mejora.

La formalización de la evaluación toma varias direcciones. La relativa solidez de la metodología del ACB y su capacidad de integración lleva a intentos de incorporar tanto elementos medioambientales como sociales. Esto exige la cuantificación de los impactos correspondientes y la determinación de un valor monetario para esos impactos.

Al ganar en importancia los elementos ambientales, y formalizarse autónomamente la metodología de Evaluación de Impacto Ambiental, se desarrollan sistemas de presentación de resultados que acaban cristalizando en el análisis multicriterio (AMC). Si bien en algunos casos se establecen sistemas de valoración y ponderación de los diferentes criterios que intentan resumir los resultados en un único indicador, es más común el reconocer el carácter arbitrario de esos intentos de ponderación, y preferir la presentación de los resultados para cada criterio de manera independiente.

A diferencia de la evaluación de tipo económico o ambiental, la evaluación de los efectos sobre la «ordenación del territorio» está lejos de haberse formalizado. Dentro de la ordenación del territorio se encuentran buena parte de los efectos indirectos o, en la terminología internacional actual wider impacts, que no pueden considerarse como propiamente ambientales. En parte, estos efectos se abordan con herramientas de evaluación económica: es el caso de los efectos sobre el empleo o sobre el PIB. El consenso académico aconseja evitar el obtener valores de referencia trasladables de un proyecto a otro, y en su lugar, se recomienda elaborar estudios ad hoc, pero no de manera sistemática, sino sólo cuando realmente haya buenas razones para pensar que estos efectos pueden ser significativos (ITF, 2008). La realidad en España es más bien la contraria: se aplican con escaso espíritu crítico ratios y elasticidades de un estudio a otro para estimar con indudable osadía el número de nuevos empleos o el incremento en el crecimiento del PIB en la región donde se implantará la infraestructura.

\footnotetext{
${ }^{2}$ El Ministerio de Fomento, como «órgano promotor» del proyecto, corre en este momento el riesgo cierto de adoptar un papel impulsor, a riesgo de la deseable neutralidad en la evaluación del proyecto.
} 
Los efectos sobre el «territorio» se convierten así en la parte más incierta de la evaluación. Esta evidente debilidad se acentúa cuando en el diálogo de concertación participan con un peso relevante agentes territoriales (regiones, ciudades...), pues los efectos indirectos suelen convertirse entonces en un factor de enorme importancia en la movilización de esos agentes y, en definitiva, en las verdaderas razones de justificación de la actuación.

Una correcta formalización de la toma de decisiones exige la identificación de los agentes que pueden intervenir en cada momento, del tipo de interacción que establecen con la administración de transporte (desde el acceso a la información hasta la capacidad de negociación pasando por situaciones intermedias) y de las circunstancias que deben concurrir para poder adoptar una determinada decisión sobre cada actuación. En España, el proceso se encuentra formalizado claramente en las fases finales de definición de la actuación, cuando entra en juego la normativa de evaluación ambiental de proyectos, mientras que las fases previas, de planificación, carecen en buena parte de procedimientos reglados para articular la participación, e incluso para definir qué tipo de información y de interacción con otros agentes es precisa antes de tomar una decisión (por ejemplo, para incorporar una nueva actuación a un plan ya aprobado).

\section{El sistema actual de evaluación de proyectos y toma de decisiones en el Ministerio de Fomento}

En este apartado se explorará con algún detalle el sistema de evaluación de proyectos y de toma de decisiones dentro del ámbito de competencias del Ministerio de Fomento, con particular atención a tres cuestiones:

- En primer lugar, qué herramientas de evaluación de proyecto se están utilizando, y cómo se ponen en relación entre sí; ¿es precisa una mejora en estas herramientas técnicas, y si es así, en qué dirección?

- En segundo lugar, cómo se ha formalizado el proceso de toma de decisiones. ¿Satisface las exigencias de legitimidad? ¿En qué medida choca con la «racionalidad»?

- Finalmente, qué perspectivas de evolución se plantean en el proceso de toma de decisiones para avanzar en las dos direcciones anteriores (legitimidad y racionalidad), y cuál podría ser la contribución de las herramientas de evaluación de proyectos.

\subsection{La evolución del sistema de toma de decisiones en las fases de planificación y proyecto}

Los procesos de tramitación de las actuaciones en infraestructuras de transporte se encuentran definidos en las leyes sectoriales correspondientes y en la normativa 
que las desarrolla: la ley 25/1988 de carreteras, la ley 39/2003, del sector ferroviario, la ley 27/1992 y 62/1997 de puertos del Estado y de la marina mercante, al que se añade la ley 48/2003, de régimen económico y de prestación de servicios de los puertos de interés general. En el ámbito aeroportuario, las funciones de planificación de infraestructuras aeroportuarias corresponden a AENA, y se desarrollan a través de Planes Directores para cada aeropuerto, cuya necesidad viene recogida en el artículo 166 de la Ley 13/1996, de 30 de diciembre, de Medidas Fiscales, Administrativas y del Orden Social, desarrollado por el Real Decreto 2591/1998, de 4 de diciembre, sobre la Ordenación de los Aeropuertos de Interés General y su Zona de Servicio. Quedan así diferenciadas dos situaciones: en puertos y aeropuertos, existe el llamado plan director de cada puerto o aeropuerto, que establece las eventuales inversiones a realizar a corto y medio plazo, e incluye un análisis de demanda y económico-financiero para el conjunto del plan. En carreteras y ferrocarriles, la legislación es poco precisa sobre la planificación y sólo al llegar al llamado estudio informativo (que se refiere a una actuación concreta) detalla el contenido mínimo del documento a elaborar, que incluye el análisis de alternativas, la realización de estudios de impacto ambiental, y el proceso de información pública.

Esta situación permite afirmar que el sistema de evaluación de proyectos y de toma de decisiones en España dirige sus energías al análisis de cada actuación concreta por separado, y está escasamente condicionado por los planes de carácter más general, que ni son obligatorios ni cuentan con capacidad legal para imponer sus directrices. Por sencillez, expositiva, nos referimos a estos estudios como el estudio informativo, sabiendo que en el caso de puertos y aeropuertos reciben otro tipo de denominaciones. El estudio informativo (definido en cuanto a su alcance y contenidos tanto en la ley de carreteras como en la ley de ferrocarriles) contiene:

- La definición de diversas alternativas para el proyecto.

- La evaluación de esas alternativas mediante un análisis coste-beneficio y un análisis multicriterio.

- El estudio de impacto ambiental.

- La propuesta en cuanto a la selección de la alternativa más adecuada, basada en el análisis multicriterio.

El estudio informativo y su estudio de impacto ambiental se someten a información pública. La Declaración de Impacto Ambiental (DIA), que concluye el proceso de evaluación del estudio informativo, implica la aprobación de la solución propuesta por el estudio informativo o bien su rechazo motivado. A partir de esa declaración, el Ministerio de Fomento puede aprobar definitivamente el estudio informativo, salvo que la DIA haya concluido con el rechazo del estudio; en ese caso, el Ministerio de Fomento puede abandonar la actuación, elaborar un nuevo estudio con otras alternativas o elevar la aprobación del estudio rechazado por la DIA al Consejo de Ministros.

En definitiva, el estudio informativo y su DIA son los documentos en los que la administración (tanto como autoridad responsable de transporte como ambiental) 
motiva la conveniencia de emprender un determinado proyecto basándose en consideraciones de utilidad pública, es decir, de conseguir un beneficio social que justifica el empleo de una cierta cantidad de recursos y una serie de impactos negativos, convenientemente evaluados.

El estudio informativo se refiere habitualmente a actuaciones de una cierta dimensión, que después pueden articularse en varios proyectos constructivos, pero acotados: no se realizan estudios informativos por ejemplo, de todo un nuevo corredor de transporte, sino que ese corredor se divide previamente en varios tramos.

El estudio informativo ocupa así una posición central en la evaluación de proyectos, y en la relación entre dicha evaluación «técnica» y la toma de decisión. Se analiza esta relación en el apartado siguiente. En este momento, lo que interesa es cómo se articula el proceso de toma de decisiones «aguas arriba y aguas abajo» del propio estudio informativo, es decir:

- «Aguas arriba», cómo se identifican las actuaciones que van a ser objeto de un estudio informativo.

- «Aguas abajo», cómo se traducen las conclusiones de un estudio informativo en la programación y ejecución del proyecto correspondiente.

$\mathrm{El}$ análisis del proceso «aguas arriba» es, posiblemente, el más interesante para los objetivos de este trabajo. En España no existe una formalización del sistema de planificación de transporte. Esto es una anomalía relativa con respecto a los países de nuestro entorno: mientras en algunas casos el proceso de planificación está claramente establecido en la legislación (Alemania) o exige una participación activa del parlamento (Países Bajos), en otros el sistema de planificación se «reinventa» periódicamente en función de las prioridades políticas ${ }^{3}$ o se liga estrechamente lal ciclo político como mero «programa de gobierno» (caso de Italia).

Al no existir en España un procedimiento normalizado, el resultado es que las actuaciones objeto de un estudio informativo, aunque pueden estar identificadas en algún tipo de planificación superior, pueden también surgir de manera «espontánea», bien como resultado de la interacción de los distintos agentes con los decisores políticos, bien por identificación de necesidades, más o menos justificadas, desde los propios servicios técnicos ministeriales. En todos los casos hay una cierta justificación técnica, aportada por esos impulsores iniciales, que si bien carece de uniformidad, incorpora tres tipos de consideraciones:

- Las ligadas a la demanda de transporte.

- Las vinculadas al medio ambiente.

\footnotetext{
${ }^{3}$ Como en Francia, donde se ha pasado sucesivamente por una planificación basada en la legislación «Schémas des Services de Transports») a finales de los años 90, seguida por otra con un fuerte componente de concertación interinstitucional (el «Comité Interministériel d'Aménagement et de Développement du Territoire, CIADT» durante la primera parte de esta década, para llegar, actualmente, a un sistema basado en los objetivos de desarrollo sostenible con la llamada «Ley Grenelle».
} 
- Las relacionadas con los efectos sobre el desarrollo económico del territorio afectado por la infraestructura, y que denominaremos como «pseudo-territoriales». Estos son en buena medida los efectos indirectos o wider economic impacts ya citados anteriormente.

La experiencia es que la existencia formal de algún tipo de plan ha sido útil para limitar el número de estas iniciativas más o menos «espontáneas», y para realizar una evaluación más homogénea de los proyectos.

Al no existir una formalización del sistema de planificación, su papel dentro de la toma de decisiones no ha estado claro, ni tampoco los instrumentos técnicos para identificar y evaluar los proyectos a incluir en el plan. Es ilustrativo el analizar brevemente la evolución de la toma de decisiones más reciente:

- Los planes de los años 80 (Plan de Transporte Ferroviario, PTF, y Plan General de Carreteras, PGC) siguen una fuerte «ortodoxia» técnica en cuanto a la evaluación, si bien con un componente casi exclusivamente económico, focalizado en la valoración de los tráficos esperados. Esto se formaliza lógicamente en un ACB convencional. No se realiza en aquellos planes ningún tipo de evaluación ambiental ${ }^{4}$. Desde el punto de vista de la toma de decisiones, el PGC viene amparado por la Ley de Carreteras de 1974 y, de hecho, el desarrollo del PGC servirá para consolidar el sistema todavía vigente de tramitación de proyectos, articulado en torno al Estudio Informativo; en contraste, el PTF no responde a ningún requerimiento normativo específico. El resultado son propuestas de actuación muy ajustadas a la lógica económica (hoy diríamos a los resultados del ACB) y con muy escaso nivel de legitimación (en el sentido de no haber generado apenas proceso alguno de diálogo y concertación). El desinterés en aquel momento por estos planes desde las Comunidades Autónomas, la clase política y los medios de comunicación, sobre todo si se compara con la situación actual, estuvo probablemente justificado por el escepticismo entonces dominante sobre la planificación, después de que los planes de principios los años 70 hubieran sido víctimas, sin excepción, de la crisis económica.

- En los años 90, el Plan Director de Infraestructuras (PDI) realiza un notable esfuerzo de evaluación. Además de la evaluación de demanda y de los ACB, se realiza un estudio de tipo territorial, que intenta establecer la relación entre el futuro sistema de transporte y un modelo territorial para el conjunto del país: algo inédito desde que la Constitución de 1978 hubiera establecido las compe-

\footnotetext{
${ }^{4}$ En aquel momento España no estaba sometida a la normativa europea sobre evaluación ambiental, por otra parte todavía poco desarrollada: la directiva 85/337/CE, de evaluación de impacto ambiental (EIA), se encontraba en elaboración, de manera que la única referencia ambiental era la Directiva 79/409/CE, conocida como directiva «aves», que es el primer antecedente sobre la preocupación del impacto de las infraestructuras sobre el medio ambiente. La tradición era además de escasa preocupación por estas cuestiones, de manera que tras la incorporación a las Comunidades Europeas, la aplicación que España comienza a hacer de la directiva de EIA no es precisamente ambiciosa.
} 
tencias de ordenación territorial en el ámbito autonómico. Menos ambicioso resulta el estudio ambiental (debe recordarse que aún no existía el concepto de una evaluación ambiental estratégica y que sólo la directiva 79/409/CE establecía algunas exigencias, por lo que la evaluación ambiental se posponía a la fase de elaboración de los proyectos concretos). Más relevante es el trabajo de concertación: el PDI tuvo un proceso de redacción relativamente rápido (199192) seguido de un proceso de concertación largo (si bien nunca tuvo proceso alguno de participación pública), hasta su presentación en el Congreso de los Diputados en 1994.

- Al PDI sigue un proceso de progresiva pérdida de prestigio de la planificación, no limitado a la política nacional de transporte, sino también evidente en los ámbitos autonómico y local. Se entra en una fase de «planificación abierta», en el que cualquier momento es adecuado para plantear nuevas actuaciones, que adquieren el mismo nivel de legitimidad que las consideradas en el PDI. Este sistema tiene efectos diversos: desde la perspectiva de la toma de decisiones, refuerza cada vez más el papel de la concertación entre administraciones, si bien de una manera escasamente formalizada: las administraciones autonómicas y locales comienzan a establecer sus propios listados de necesidades en infraestructuras de transporte de competencia estatal, y a intentar negociar su ejecución con el Ministerio de Fomento. Distintos grupos económicos, y singularmente las empresas constructoras, comienzan a actuar de manera similar, «proponiendo» al Ministerio de Fomento nuevas actuaciones, al amparo de la pretendida impulsión de la inversión privada en infraestructuras. El sistema de participación que se configura resulta enormemente asimétrico: abierto casi en permanencia a los agentes institucionales y a los grandes intereses económicos, y sumamente opaco e inaccesible para el resto de la sociedad civil.

Un elemento de interés de esta época es la importancia creciente que adquieren las infraestructuras de transporte dentro de los procesos electorales. Hasta principios de los años 90, el punto de partida para la identificación de actuaciones habría residido en la «racionalidad técnica», y el proceso de diálogo con otras administraciones se habría desarrollado posteriormente, con el propósito de facilitar la realización material del proyecto, dotándole de una «legitimidad» en cierto modo ex post, ahora el proceso se invierte: la identificación de una actuación se produce de manera confusa, y aparece como promesa electoral que se convierte en caso de éxito electoral en programa de gobierno, dando por supuesto y haciendo secundaria la «racionalidad técnica». La evaluación de proyectos se mantiene, pero en buena medida a posteriori de la decisión de ejecución de proyectos, buscando la solución más racional dentro del respeto a las promesas y compromisos pactados.

- El regreso formal a la planificación se produce en 2004, e inicialmente se plantea una ambiciosa agenda: por una parte, reforzar el sistema de evaluación previo a la toma de decisiones; por otra, canalizar y formalizar los procesos de diálogo y concertación, y abrirlos al conjunto de la sociedad civil; finalmente, 
clarificar y racionalizar el proceso de toma de decisiones. Aquí el futuro PEIT contaba con un aliado prometedor: la directiva europea de evaluación ambiental estratégica (2001/42/CE), que debía entrar en vigor ese mismo año.

El PEIT es el primer plan de transporte que se somete a un proceso formalizado de participación pública y aprobación, y en este sentido supone un modesto avance en la formalización del proceso de concertación y participación. Sin embargo, no conseguirá consolidar esta práctica, y los sucesivos «planes» que ha preparado posteriormente el Ministerio han vuelto a la práctica de evitar la participación ciudadana. Los resultados de aquel proceso pusieron de manifiesto que el interés de la mayor parte de los agentes continuaba limitándose al impulso de nuevas actuaciones en infraestructuras, lo que indudablemente debilitó la voluntad de reforma progresiva del sistema propuesta por el plan.

Las consecuencias de esa «toma de decisión» que es la aprobación del PEIT por el consejo de ministros en julio de 2005 para las diversas actuaciones no se entienden de la misma manera por parte de los diversos agentes: desde la perspectiva técnica, las actuaciones identificadas en los mapas del PEIT pasaban a una fase posterior de análisis más detallado, los denominados planes sectoriales (MFOM, 2004: 161); desde la perspectiva de los agentes institucionales territoriales, la ejecución de esas actuaciones se considera como decidida: las etapas siguientes, de redacción y aprobación de proyectos, no podían considerarse más que como trámites administrativos que no podrían poner en cuestión la ejecución la actuación. En realidad, los mapas incluían actuaciones con un grado muy diverso de maduración: desde proyectos en ejecución, o con proyecto constructivo ya redactado y aprobado hasta nuevas propuestas que habían surgido dentro del proceso de concertación. El PEIT resultó relativamente exitoso en impedir que nuevas propuestas continuaran incorporándose al listado de actuaciones futuras del Ministerio de Fomento, pero acabó de hecho otorgando a las actuaciones contenidas en sus mapas una racionalidad que no podía justificarse más que en contados casos a partir de los someros estudios de evaluación hasta ese momento realizados.

\subsection{Las relaciones entre instrumentos de evaluación técnica y toma de decisiones en el caso del PEIT}

La reciente experiencia del PEIT puede servir para identificar y analizar las interacciones que se establecen entre la racionalidad técnica y la legitimación política y que subyacen en todo el proceso de toma de decisiones. Los instrumentos de evaluación del PEIT consistieron en un análisis coste beneficio somero y una evaluación ambiental estratégica. Se realizó además, un estudio de accesibilidad como aproximación a los efectos esperados sobre la cohesión territorial.

El análisis coste beneficio puso de manifiesto la escasa rentabilidad socioeconómica de la mayor parte de las actuaciones que se estaban planteando, incluyendo 
aquellas que el Ministerio de Fomento tenía ya relativamente avanzadas. A título de ejemplo, las actuaciones en la red ferroviaria de altas prestaciones resultaban con ratios beneficio/coste inferiores a la unidad para la mayor parte de los ejes considerados.

El análisis de accesibilidad pone de manifiesto las importantes limitaciones del sistema ferroviario existente en la mayor parte del territorio y las importantes ganancias potenciales de accesibilidad que se pueden conseguir. Hay que señalar, no obstante, que los indicadores de accesiblidad no incorporan ningún peso para el territorio donde se está calculando la accesibilidad, por lo que una ganancia importante de accesibilidad no significa necesariamente que haya una demanda importante de movilidad. Otra limitación importante del estudio es el no integrar ningún parámetro relacionado con la frecuencia esperada de los servicios (que a su vez está necesariamente vinculado a la demanda): una frecuencia de servicio baja significa una accesibilidad también baja, aunque la infraestructura permita tiempos de recorrido muy reducidos (Gráficos 1 y 2 y véanse mapas de accesibilidad ferroviaria en Ministerio de Fomento, 2005).

\section{GRÁFICO 1}

\section{ACCESIBILIDAD DEL TERRITORIO POR FERROCARRIL:} SITUACIÓN 2004

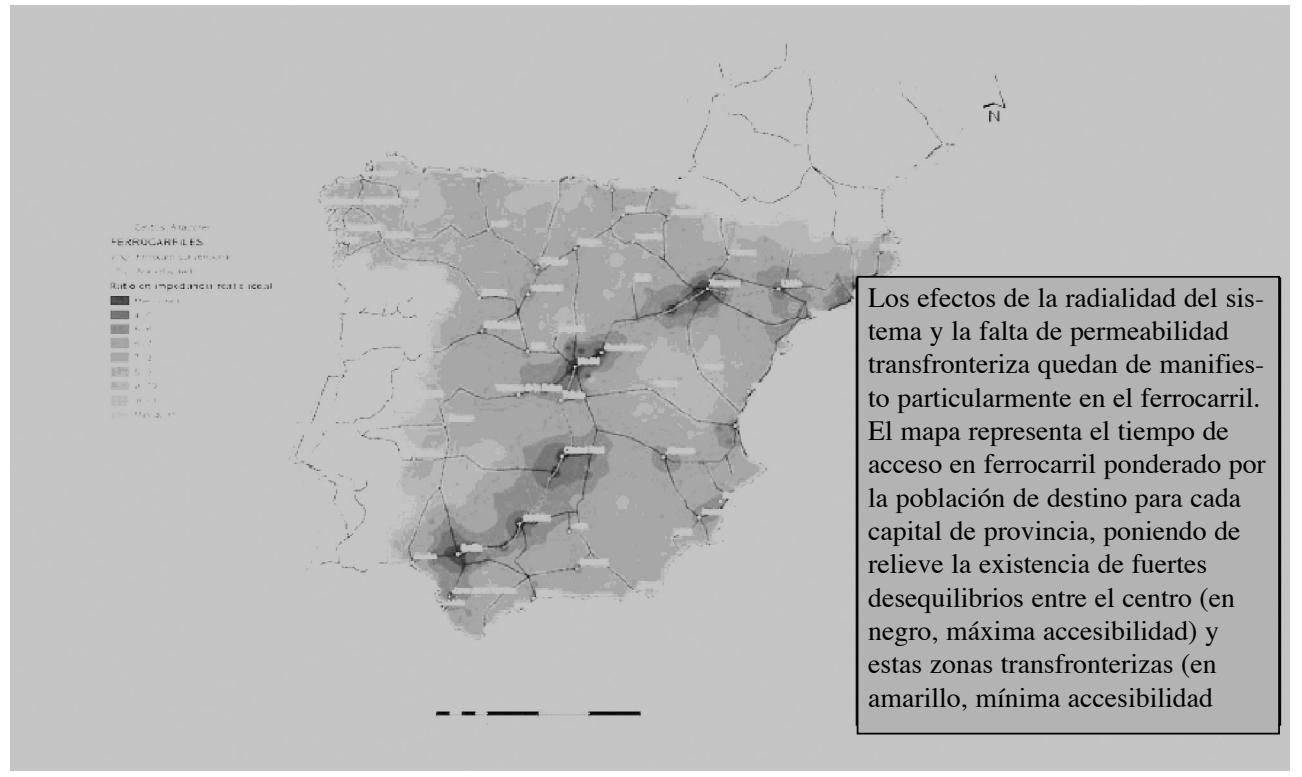




\section{GRÁFICO 2}

ACCESIBILIDAD DEL TERRITORIO POR FERROCARRIL: SITUACIÓN 2020 (SI SE REALIZAN TODAS LAS ACTUACIONES)

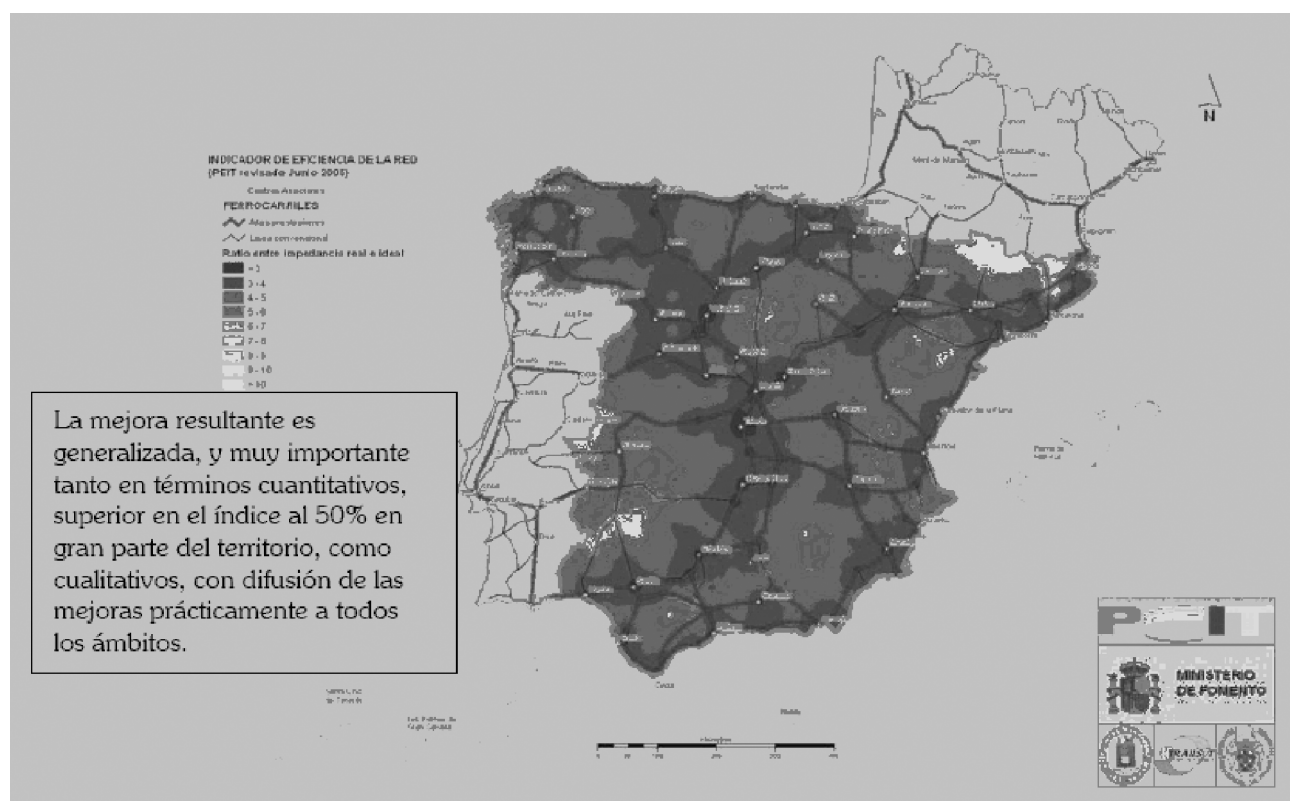

La Evaluación Ambiental Estratégica se acomoda al proceso de toma de decisión «en cascada» con el que el PEIT pretende formalizar el proceso de planificación, cubriendo el vacío existente hasta la evaluación de actuaciones en los estudios informativos. (Ministerio de Fomento, 2004: 19 y 20). De esta forma, analiza con cierto detalle la coherencia e implicaciones ambientales de las directrices establecidas por el PEIT, y propone aplicar una plantilla de análisis de «compatibilidad ambiental estratégica de las actuaciones del PEIT para cada una de las actuaciones concretas, análisis que se realizaría materialmente durante la elaboración de los planes sectoriales que deberían desarrollar el PEIT. (Ministerio de Fomento, 2004: 151) (véase cuadro de texto).

Con ello, la EAE de alguna manera relativizaba la importancia de las propuestas concretas que aparecían en el apartado de «prioridades y ámbitos de actuación sectorial» del PEIT, incluidas las actuaciones en infraestructuras. La lógica era que la escala adecuada de trabajo para el análisis de proyectos era la de la fase siguiente, es decir, los planes sectoriales, y que el cuestionario de compatibilidad ambiental estratégica serviría como un elemento previo para, junto con la información procedente del ACB y del análisis territorial, incorporar cada actuación al plan sectorial correspondiente. 
Aquí detecta el ISA un riesgo considerable. El riesgo radica en que la incorporación de la actuación o ámbito de actuación al PEIT se considere de facto una aprobación de la misma, sin que medie la realización de su análisis de compatibilidad ambiental estratégica desarrollada en el cuestionario ACAE. Y que luego dada su inclusión en el PEIT no se considere necesario su evaluación ambiental a nivel del plan sectorial correspondiente. Ello impediría de facto la realización en cascada de los criterios estratégicos del PEIT. (Ministerio de Fomento, 2004: 161).

De hecho, podría decirse que este riesgo se ha materializado cinco años después. La ausencia de planes sectoriales, y de procesos formalizados de revisión del propio PEIT han impedido profundizar en la evaluación individualizada de las actuaciones. Sea como causa o como consecuencia de lo anterior, el proceso de toma de decisiones no ha llegado a modificarse significativamente, ni en lo referente a la transparencia sobre el resultado de la interacción con otros agentes, ni en cuanto a la apertura del diálogo a nuevos interlocutores. La multiplicación de todo tipo de convenios con las comunidades autónomas durante estos años (bien que en general, limitadas a actuaciones incluidas en los mapas del PEIT) ha continuado consolidando un modelo de toma de decisiones basado en la concertación, pero cerrado a los agentes no institucionales.

\subsection{El sistema de toma de decisiones como consecuencia de la disponibilidad de recursos financieros}

La reciente crisis económica y la finalización de buena parte de los recursos procedentes del Fondo de Cohesión de la UE a partir de 2013 invitan a reflexionar sobre el papel que puede haber desempeñado en el sistema de toma de decisiones la cuantiosa financiación europea de las infraestructuras de transporte con la que España ha contado desde mediados de los años 80. Puede argumentarse que en el modelo de toma de decisiones que se ha consolidado puede haber influido la disponibilidad de considerables recursos financieros de la UE. La existencia de estos fondos genera una gran demanda de proyectos de transporte desde finales de los años 80 . Es sorprendente que el país no haya decidido en ningún momento un cambio sustancial de estrategia de empleo de los fondos hacia otras políticas públicas a pesar de la evidencia de que la rentabilidad socioeconómica de las actuaciones seleccionadas disminuía progresivamente ${ }^{5}$. No es de extrañar que se produzca en estos 20 años un descenso apreciable en los niveles de demanda/tráfico esperado en los proyectos, descenso que se trata de compensar con referencias cada vez más estereotipadas a todo tipo de beneficios indirectos. Desde la perspectiva de la toma de decisiones, la necesidad de absorber estos fondos europeos genera un cierto desinterés por los

\footnotetext{
5 A pesar de que en algún momento (por ejemplo a mediados de los años 90) se plantean iniciativas que no llegan a cuajar, por ejemplo, de apoyo a políticas centradas en las ciudades, existentes en la mayor parte de Europa
} 
resultados económicos del ACB y un creciente peso del diálogo interinstitucional. Ahora bien, los agentes institucionales asocian el éxito de su gestión con la ejecución de nuevos proyectos; el diálogo se centra entonces en identificar los proyectos que concilian las expectativas de reconocimiento de unos y otros por parte de la opinión pública. Esto explicaría, por ejemplo, la estrategia de realizar tramos parciales de las grandes obras lineales en varias partes del territorio a la vez, en lugar de concentrar los recursos en un número más limitado de itinerarios completos para acelerar en lo posible su puesta en marcha: sirvan como ejemplo de una práctica generalizada el caso de la autopista A-8 (el primer tramo- de peaje- es de principios de los años 70 y algunos tramos se encuentran todavía en ejecución) o la línea ferroviaria de alta velocidad Madrid-Barcelona (iniciada a mediados de los años 90 y finalizada a principios de 2008).

Las mayores carencias del modelo de toma de decisiones parecen ser su apertura y transparencia, sobre todo en las fases iniciales de la planificación hasta la elaboración del estudio informativo. El hecho de que se produzca un intenso diálogo institucional, en buena parte seguido e incluso amplificado por los medios de comunicación, parece contradictorio con esta afirmación. Sin embargo, este tipo de concertación tiene el riesgo de ser sesgado por varias razones:

- Acceso dispar a la información disponible por parte de los agentes que intervienen.

- Acceso restringido, donde no está prevista la participación de los agentes no institucionales. Se supone que estos agentes sociales y económicos deben buscar el acceso al debate de manera indirecta, intentando influir sobre los agentes institucionales que sí tienen acceso.

- Paradójicamente, aunque el diálogo sea un diálogo entre instituciones, carece de reglas precisas, es decir, de una formalización: las reglas del juego no están establecidas, incluido en lo referente al proceso de adopción de acuerdos. Incluso podría decirse que los elementos relevantes para el debate, es decir, el tipo de «buenas razones y argumentos» que los interlocutores se deben para poder dialogar y convenir, no están identificados siquiera de manera aproximada, por lo que el proceso de negociación y de decisión está prácticamente inerme ante todo tipo de consideraciones puramente coyunturales.

- La ausencia de un árbitro o mediador en el proceso, al estilo de los responsables de las encuestas de utilidad pública en Reino Unido, Francia y otros países.

Todas estas circunstancias establecen un campo de juego nada neutral, prácticamente cerrado a los agentes sociales con menos recursos. Con todo, la principal limitación de este proceso está siendo su incapacidad de incorporar elementos de gobernanza modernos, como son la Evaluación Ambiental Estratégica, el fortalecimiento de la participación pública en las tomas de decisiones que tienen un particular efecto sobre el medio ambiente o el seguimiento de las políticas públicas respecto de objetivos y no simplemente de ejecución presupuestaria. 
El proceso de decisión «aguas abajo» del estudio informativo presenta unas condiciones de partida diferentes, ya que los procedimientos administrativos están establecidos con claridad hasta llegar a la licitación y ejecución del proyecto. El procedimiento deja, lógicamente, amplio margen en cuanto a los ritmos de avance, y se hace frecuentemente preciso el diálogo con otros agentes para resolver las más variadas cuestiones en la definición del proyecto.

En cierta forma, la gestión de este proceso «aguas abajo» se convierte en un instrumento fundamental para compensar la ausencia de una programación explícita de las actuaciones en el momento de la planificación. Esta cuestión no es baladí: téngase en cuenta que, aparentemente, uno de los obstáculos más relevantes para la aprobación de los planes sectoriales que debían concretar y precisar las actuaciones del PEIT ha sido el riesgo percibido por los responsables del Ministerio de que al concretar las actuaciones a realizar en cada uno de los cuatrienios hasta llegar al horizonte 2020 del PEIT, los agentes institucionales (principalmente territoriales) consideraran inadmisible el hecho de que algunas de las actuaciones de su interés quedaran formalmente fuera de la programación más inmediata.

Queda de manifiesto así la difícil conciliación entre la lógica técnica y la política. La primera considera necesario definir con antelación la programación de inversiones prevista, mientras que la segunda desea contar con un amplio margen de maniobra (fruto de una cierta indefinición) para poder articular un consenso suficiente de legitimación de su gestión. El problema es si esta actitud resulta compatible con las exigencias de transparencia en la gestión de una política pública. Incluso desde un planteamiento de pura eficiencia, resulta difícil justificar cómo una ausencia de programación puede resultar beneficiosa desde las dos perspectivas: desde la racionalidad, porque transmite la impresión de que no se cuenta con herramientas técnicas capaces de ayudar a identificar las prioridades; desde la legitimidad, porque parece albergar no sólo una ausencia de criterios, sino también un deseo de opacidad en cuanto a la motivación de sus decisiones.

\subsection{Las herramientas de evaluación de proyectos}

$\mathrm{Al}$ describir en un apartado anterior el proceso de toma de decisiones, se identificaron las principales herramientas de evaluación disponibles, señalando que su empleo principal se encuentra en la fase del estudio informativo. En esa fase, la evaluación de proyectos se realiza desde dos perspectivas: la evaluación socioeconómica, basada en el ACB y la evaluación ambiental, realizada en el marco del estudio de impacto ambiental. Los resultados de ambas se integran frecuentemente mediante una metodología multicriterio. Junto a estas técnicas de evaluación, es frecuente encontrar otras, interesadas en los llamados efectos territoriales. A continuación, se analizan dichas herramientas con más detalle.

Cada modo de transporte ha adaptado de una manera particular la metodología del análisis coste-beneficio a sus circunstancias particulares. Aunque metodológica- 
mente son similares, estas adaptaciones difieren tanto en los elementos incluidos en el análisis como en los valores monetarios que asignan a algunas de las variables. Una de las principales diferencias se produce en la valoración del tiempo de los usuarios.

El ACB parece haberse consolidado como una metodología útil para la evaluación de alternativas dentro de una actuación individual, pero la identificación y selección de estas actuaciones precisa de instrumentos de análisis de otro tipo, aptos para la consideración del conjunto del sistema de transporte. Esta es de hecho la situación en España, y a esta conclusión parece haberse llegado también en Europa, si se toma como referencia la reciente revisión metodológica realizada para la planificación de la red transeuropea de transporte (European Commission, 2010).

El interés por los efectos territoriales ha llevado a la proliferación de los llamados estudios de accesibilidad. Estos estudios ya están presentes en los años 80 y 90 en el Plan General de Carreteras y en el PDI. Conocen sin embargo un importante impulso en la última década, e indefectiblemente concluyen en la consecución de mejoras considerables en la accesibilidad que, con escasa justificación, asocian con el refuerzo de la cohesión territorial y el desarrollo (véase por ejemplo, Gonzalo Orden, 2008; López Suárez, 2009; Gutiérrez Puebla, 2006).

Un elemento común en estos estudios es la utilización de indicadores de accesibilidad es que en el cálculo del indicador asociado a cada unidad territorial suele prescindirse de las características demográficas y económicas de dicha unidad territorial que van a caracterizar, aunque sí puedan tenerse en cuenta en el cálculo las características del resto de unidades territoriales. Así, por ejemplo, la accesibilidad de una provincia se calcula a partir del tiempo de viaje a las demás provincias, ponderado por la población de cada una de esas otras provincias, pero no se incluye en el cálculo la población en la provincia en la que se calcula la accesibilidad. Lo mismo ocurre si se toma como parámetro para el cálculo el empleo o el PIB. De esta manera, se está prescindiendo de un dato clave para la planificación de transporte: la mayor o menor población o actividad económica está fuertemente vinculada a la demanda de transporte, y ayuda a identificar dónde hay una demanda suficiente de movilidad para crear o no nuevas infraestructuras. Al prescindir de esos parámetros en los indicadores de accesibilidad, el resultado tiende a ser una visión «homogeneizadora» del territorio, que destaca unos pretendidos déficits o carencias de accesibilidad sin matizar sus razones, como podrían ser la escasa población o actividad económica, o las barreras naturales costosas de franquear (gráficos 1 y 2).

El atractivo -y riesgo- de los indicadores de accesibilidad es su capacidad de ofrecer una imagen sumamente intuitiva de mejora del territorio con los proyectos propuestos. A pesar de la reconocida dificultad teórica de valoración de los efectos territoriales (ECMT, 2004), la tentación es habitualmente demasiado fuerte como para evitar que esos estudios de accesibilidad den un paso más para concluir- con escaso rigor- que existe una relación directa entre esa mejora de accesibilidad y el crecimiento de variables socioeconómicas como el empleo o el PIB. 
La dificultad y controversia sobre los impactos territoriales quizá estén relacionadas con la ausencia, o al menos falta de explicitación suficiente, de las estrategias de ordenación del territorio y de desarrollo regional en los territorios afectados. Hay que recordar que, a partir de 1978, la competencia legal sobre la Ordenación del territorio se establece en cada Comunidad Autónoma, lo que ciertamente no favorece y, en la práctica lleva a abandonar, una perspectiva territorial para el conjunto del Estado. Pero se conserva una visión del sistema de transporte como elemento vertebrador del territorio que cuenta con una enorme tradición en España: piénsese por ejemplo en el despliegue de la red ferroviaria en la segunda mitad del XIX, fundamentado en consideraciones territoriales más que económicas.

Desde ese interés por los efectos territoriales de las infraestructuras de transporte se emprenden los primeros estudios de accesibilidad, en el Plan General de Carreteras primero y después en el PDI, estudios que se amplían posteriormente al conjunto de las primeras propuestas de red transpeuropea de transporte, y la administración española interviene activamente en los debates sobre la futura «Estrategia Territorial Europea» durante el período 1994-1999 defendiendo un «acceso equivalente a las infraestructuras» (sic). Esta estrategia europea propone la «introducción de los estudios de impacto territorial como instrumento de evaluación espacial de los grandes proyectos de infraestructuras, en particular en el ámbito de los transportes» (Comité de Desarrollo Territorial, 1999: 30).

El PDI resulta particularmente ilustrativo de la mezcla de interés e impotencia desde la que los planificadores de transporte en el ámbito nacional abordan la cuestión territorial. En el PDI se distingue entre tres objetivos territoriales (mejorar la eficiencia del territorio, desarrollar la equidad espacial y social y poner en valor el medio ambiente y el patrimonio histórico y cultural), y se asocia a cada uno de ellos un tipo diferente de actuación en transporte. El primer tipo de actuaciones agrupa las infraestructuras de mayor capacidad, generalmente situadas en las partes más dinámicas del territorio; las segundas requieren la colaboración estrecha con las otras administraciones para apoyar el potencial de desarrollo del resto del territorio; las terceras pretenden actuar en las áreas con mayores riesgos ambientales desde la perspectiva del transporte, en particular en las ciudades. Esta parte del documento termina recordando la necesidad de adoptar «medidas complementarias» coordinadamente entre las administraciones públicas para lograr mayores efectos (MOPTMA, 1994: 58 y 59).

Es inevitable plantearse la cuestión sobre las razones que justifican este intento por parte de la planificación sectorial de transporte por convertirse en elemento vertebrador de una política territorial. Una respuesta puede encontrarse en la ausencia (o al menos debilidad) de estrategias territoriales explícitas en el ámbito autonómico, que es donde reside esta competencia. Efectivamente, la situación de la ordenación territorial es sumamente heterogénea en el conjunto del país, de manera que es difícil encontrar coherencia en la imagen de conjunto: algunas comunidades autónomas han prescindido de la formalización de su política de ordenación territorial, 
otras han contado en algunos momentos con planes territoriales y, finalmente, sólo una pocas han llegado a consolidar y mantener en el tiempo una tradición de planeamiento territorial. Desgraciadamente, incluso cuando existe una planificación territorial formalizada, esto puede no ser suficiente si esa planificación no cuenta con los mecanismos adecuados de inserción con otras políticas sectoriales- y en particular transporte- en el propio ámbito autonómico. La imagen de conjunto es la de una relación débil, en la que se produce una mera yuxtaposición de discursos sectoriales más que una auténtica interacción.

La consolidación de una visión del territorio y de una estrategia de desarrollo territorial podría considerarse como un factor de estabilidad y racionalidad, con fuerte influencia en la toma de posición de las administraciones autonómicas en el proceso de concertación institucional. Su ausencia podría ayudar a explicar el porqué de la proliferación de posturas erráticas o de petición acrítica de nuevas inversiones en infraestructuras.

En cierta manera, puede decirse que esta situación heterogénea en la ordenación del territorio, y el fuerte componente territorial en el discurso de la planificación de transporte conduce a la consolidación de una cierta perspectiva «pseudoterritorial», en el que la ausencia de herramientas de análisis sólidas y la debilidad de la propia política territorial frente al sector del transporte, posibilita la cristalización de políticas muy alejadas de la tradición de la ordenación del territorio, ya que:

- En lugar de centrarse en las potencialidades y singularidades del territorio, busca la homogeneidad de éste, y hacen del transporte (de la accesibilidad) el mecanismo fundamental de homogeneización.

- Ignoran las medidas complementarias necesarias para el desarrollo regional, de las que en buena lógica deberían derivarse las necesidades de transporte, y no al revés.

- Tienen una escasa consideración por el valor de los recursos ambientales en el desarrollo regional, lo que a su vez allana el camino a la demanda de nuevas infraestructuras de transporte.

- No parecen capaces de trabajar de manera cooperativa en una estrategia de desarrollo regional con las políticas de otras administraciones, al limitar el diálogo interinstitucional a una negociación sobre inversiones y plazos, sin referencia a objetivos y metas compartidos.

- Resultan, finalmente, opacas, al no aportar ni con un modelo territorial claramente explicitado ni un procedimiento de concertación transparente.

Finalmente, otra herramienta de evaluación, el Estudio de Impacto Ambiental, ofrece información de distinto tipo para la toma de decisiones:

- identifica la existencia de condicionantes ambientales para la implantación del proyecto en el territorio, que eventualmente pueden llegar a desaconsejar su ejecución; 
- evalúa los impactos sobre el medio ambiente, que pueden valorarse económicamente (como efectos externos dentro del ACB) o de manera cualitativa para integrarse en un análisis multicriterio.

La EIA desemboca en la declaración de impacto ambiental (DIA) por parte de las autoridades ambientales. La DIA, en cierta manera, constituye una valoración final de las alternativas del proyecto. Las limitaciones de la evaluación de proyectos y las indeterminaciones en cuanto a las competencias respectivas de la administración de transporte y la administración ambiental ha creado una interesante situación en torno a la DIA. La administración ambiental ha intentado reaccionar de distintas maneras a una situación que ha interpretado como de rigor insuficiente en la evaluación de alternativas. En este contexto, no dejan de producirse situaciones de conflicto, como el proponer desde la autoridad ambiental la selección de una alternativa diferente, modificando los criterios y pesos de la evaluación multicriterio, o de utilizar la DIA como una herramienta de presión para incrementar las actuaciones compensatorias, o condicionar la aprobación a la realización de nuevos estudios de evaluación complementarios. Esta situación refleja, probablemente, importantes debilidades en el proceso de evaluación, como por ejemplo una cierta falta de consistencia en la metodología de los estudios de evaluación, una insuficiente integración de los objetivos medioambientales durante la concepción del proyecto, y hasta una ausencia de diálogo entre los profesionales y funcionarios de los dos sectores.

\section{Fortalezas y debilidades del sistema actual de toma de decisiones}

El sistema de toma de decisiones puede valorarse desde diferentes perspectivas complementarias (Stetcher, 2010; Innes, 2004; Healey, 1986, Forester, 2008). La primera es la de la eficiencia, que valora en qué medida las decisiones adoptadas permiten alcanzar los objetivos planteados por una política pública, y hacerlo con el menor empleo de recursos posible; la segunda es la de la legitimidad, que se cuestiona si el proceso de toma de decisiones busca como objetivo el facilitar la formación de un consenso o, al menos, incluye cauces de participación, debate y diálogo; finalmente, desde la perspectiva de formalización debe reflexionarse si la toma de decisiones ha seguido los cauces legales formales previstos para su adopción, cauces que a su vez, en un contexto democrático, deben servir para garantizar la transparencia de la decisión, es decir, para que pueda conocerse de manera explícita cuáles han sido las motivaciones y quiénes han intervenido en la toma de decisiones.

La articulación de un sistema de seguimiento de objetivos desde la planificación hasta las actuaciones concretas está lejos de ser espontánea: de hecho, requiere un considerable esfuerzo de gestión para reformar las rutinas de funcionamiento de las burocracias/servicios técnicos que gestionan las actuaciones (Stetcher, 2010). Esta puede ser una razón que explique la nula atención que ha recibido el sistema de seguimiento del sector en España (Anegón, 2009) por parte de los gestores de la 
política de transporte: si no hay una identificación satisfactoria de las relaciones entre actuaciones puntuales y objetivos, el gestor puede sentirse cómodo en una valoración sobre el nivel de ejecución alcanzado, pero difícilmente en la referencia a unos resultados obtenidos gracias a la ejecución de esas actuaciones. Esta situación no puede extrañar si, por otra parte, tampoco se produce, por parte de ningún agente significativo, una presión en torno a la consecución de los objetivos de la planificación; aun más, si en su lugar se poduce una intensa presión para la rápida ejecución de las actuaciones.

Puede argumentarse que la ejecución material de las actuaciones es en sí misma un indicador de eficiencia nada desdeñable: tanto en términos de tiempo de maduración de los proyectos (desde su planificación hasta su ejecución) como de puesta en servicio, el Ministerio de Fomento muestra una capacidad de gestión muy elevada desde hace años (Ministerio de Fomento, 2007). Esto indica que el conjunto del sistema (incluida la toma de decisiones) ha permitido controlar notablemente los numerosos riesgos asociados a la concepción y ejecución de las nuevas infraestructuras. No obstante, ya se ha señalado anteriormente que es precisamente el sistema de toma de decisiones el que favorece la dispersión de actuaciones por todo el territorio en lugar de la concentración de estas, de manera que se cuenta con obras parciales completadas, pero con escasa funcionalidad, y se alargan así los plazos de finalización para completar los grandes itinerarios.

También puede argumentarse que, en cualquier caso, los objetivos de la política de transporte suelen ser excesivamente genéricos y de largo plazo como para poder vincularse con proyectos concretos. Sin embargo, esta dificultad podría abordarse incluyendo, dentro de la evaluación de cada actuación, al menos un análisis explícito de su compatibilidad con los objetivos de la política de transporte. Para ello, se cuenta ya con la experiencia de algunas iniciativas en el ámbito de la Unión Europea para consolidar una gestión de las políticas públicas mejor vinculadas a los objetivos del desarrollo sostenible. Un ejemplo significativo es el de la estrategia europea de integración de los objetivos de desarrollo sostenible en las diferentes políticas sectoriales, incluida la de transporte. Desde esta perspectiva, adoptada en $2001^{6}$, se plantea poner en marcha un proceso que permita que las decisiones, en los diferentes niveles de la política de transporte, consideren con el mismo nivel de importancia los objetivos funcionales tradicionales en el sector con los derivados de la Estrategia Europea de Desarrollo Sostenible; estos últimos se traducen, para el sector del transporte, por ejemplo, en la búsqueda del cambio modal hacia los modos con menor impacto ambiental y en la gestión del crecimiento continuado de la demanda de transporte.

Parece evidente que, sin el traslado de los objetivos al nivel de la evaluación y toma de decisiones sobre los proyectos, no resulta posible hacer operativa dicha

${ }^{6}$ Resolución del Consejo (Transportes) de la UE de 4 y 5 de abril de 2001 sobre la integración del Medio Ambiente y el Desarrollo Sostenible en la Política de Transportes. 
integración. Esto mostraría la ineficiencia del sistema de toma de decisiones, puesto que la integración se considera como un camino imprescindible para alcanzar los objetivos de desarrollo sostenible, vinculantes para todas las políticas sectoriales. La experiencia de un buen número de países en la UE prueba que esta integración es posible e incluso que se han desarrollado, instrumentos y herramientas de gestión y de evaluación para apoyar la toma de decisiones desde esta perspectiva: es el caso de la evaluación ambiental estratégica (en el ámbito de planes y programas), de la gestión ambiental (en el ámbito de las actuaciones) o de los distintos instrumentos técnicos de valoración de un número creciente de efectos de las actuaciones. Estas iniciativas están dando lugar, aparentemente, a una mejor aproximación a los objetivos de desarrollo sostenible. (ECMT, 2004; EEA, 2009).

Desde una perspectiva de legitimidad, el proceso de toma de decisiones está estrechamente ligado al tipo de participación de los distintos agentes. El derecho a la participación en las políticas públicas (y en particular en la de transporte) se ha consolidado de manera progresiva (vid Damart, 2007 por ejemplo para un análisis de la evolución en Francia), y ha estado estrechamente ligado en su origen a la atención al medio ambiente, por un lado, y a las actuaciones en medio urbano, por otro, pues inicialmente el derecho a la participación estaba acotado a las administraciones y a las personas físicas y jurídicas con un interés directo en el proyecto (ligado al derecho de propiedad en el segundo caso y a las competencias de la administración correspondiente en el primero). Es la legislación medioambiental (en el caso de España, desde finales de los años 80) la que extiende el derecho de participación a cualquier ciudadano, desde la perspectiva de que el proyecto puede tener un efecto sobre la calidad de su entorno.

El contenido y alcance de esa participación también está evolucionando rápidamente: desde una perspectiva inicial de derecho a la información hasta la lenta consolidación de procesos de diálogo, de debate e incluso de negociación entre la administración de transporte y los diversos agentes. El proceso en España no ha sido particularmente dinámico: piénsese que, aún en estos momentos, el acceso a la información está todavía limitado a los documentos que se deben someter a información pública, mientras que para el resto de documentos -que pueden haber influido en la toma de decisiones- la administración tiene una gran discrecionalidad en cuanto a facilitarla a los ciudadanos que lo soliciten ${ }^{7}$.

El hecho de que el marco normativo establezca las condiciones de aprobación de los planes y proyectos en transporte no excluye la existencia de puntos de vista divergentes. Con el término «legitimación» se hace referencia a la existencia de razones adicionales que soportan la postura de que la administración, con la solución adoptada, está trabajando efectivamente por el bien común. Parte de la legitimación viene precisamente de los instrumentos de evaluación de proyectos y de las

\footnotetext{
7 En agosto de 2010 se ha anunciado la próxima tramitación de una ley que asegure el acceso a la información de las administraciones públicas.
} 
reflexiones que anteceden a la toma de decisiones, pero estos mecanismos internos de la administración no aseguran por sí mismos, por sofisticados que sean, que el proceso acabe en una decisión conforme con el «bien común», por varias razones:

- porque no se haya tenido en cuenta información relevante, que sin embargo sí tienen algunos agentes (lo que justificaría el establecer con ellos mecanismos de diálogo, es decir, de intercambio de información);

- porque la relevancia que se da a ciertos criterios por parte de la administración pueda no ser apropiada para el caso concreto del proyecto en cuestión, o al menos así lo entiendan algunos agentes (lo que justificaría establecer mecanismos de debate, donde puedan examinarse los distintos argumentos);

- porque, finalmente, al menos algunos de estos agentes puedan estar a su vez legitimados para defender ciertos puntos de vista, en función de sus competencias administrativas (una determinada administración territorial, por ejemplo), o de otras circunstancias (por ejemplo una comunidad o colectivo que pueda verse desplazado o que vea cambiar sus condiciones de vida de manera importante por la implantación del proyecto); esto justificaría establecer mecanismos de negociación para encontrar soluciones que compatibilicen los objetivos de los distintos agentes.

En el caso de España, la situación actual sugiere que la legitimación desempeña un papel destacable en el proceso de toma de decisiones, pero que el número de agentes que participan en el proceso es extraordinariamente reducido. De hecho, se cuenta con un marco normativo en el transporte ciertamente prudente, por no decir cicatero, en términos de oportunidades formales a la participación, lo que puede ser cuando menos interpretado como una escasa preocupación por el fomento de la participación. Y, sin embargo, ya se ha descrito cómo el proceso real de toma de decisiones incluye un notable intercambio de opiniones con las otras administraciones y, en especial, la autonómica, que más allá del diálogo y del debate, se plasma finalmente en forma de convenios que recogen los resultados de la negociación (una negociación que, formalmente, no es la mayor parte de las veces necesaria desde un punto de vista puramente competencial). Pero este proceso que podría denominarse de «planificación concertada» está cerrado, casi sin excepciones, a casi cualquier otro tipo de agente.

Esto no descarta el que, en algún momento, un agente concreto pueda conseguir forzar una negociación con la administración de transporte, pero se trata ciertamente de un hecho singular. Por ejemplo, durante 2003, el interés por atraer y facilitar la inversión privada en infraestructuras de transporte llevó, en la llamada Ley de Concesiones ${ }^{8}$, a posibilitar la concesión de obras públicas «a instancia de particulares», lo que otorgaría a algunos agentes económicos la capacidad de plantear un proceso de negociación sobre una actuación a partir de un estudio de viabilidad elabo-

${ }^{8}$ Ley 13/2003, de 23 de mayo, reguladora del contrato de concesión de obras públicas. 
rado por ellos mismos, aunque la actuación en sí no estuviera contemplada dentro de las previsiones de los poderes públicos. En la práctica, sin embargo, esta situación no ha llegado a plantearse. En cambio, sí es relativamente frecuente el que otra administración se dirija al Ministerio de Fomento planteando alguna nueva propuesta de actuación, no incluida en sus planes.

Una reflexión sobre los motivos de este comportamiento de la administración de transporte puede ayudar a clarificar cuáles son los factores que dirigen el proceso de toma de decisiones. El carácter restringido del proceso a pocos agentes (y generalmente, sólo a la administración autonómica) sugiere que no existe ninguna estrategia ni posiblemente sensibilidad para materializar métodos de planificación y definición de proyecto colaborativos, pues si fuera así el proceso no conviviría con un entorno de escasísima colaboración con los otros agentes. La lógica de comportamiento de la administración de transporte puede interpretarse, más bien, desde dos perspectivas. Una funcional: la búsqueda de la aceleración y simplificación del conjunto del proceso, y otra competencial: la compensación del déficit de legitimidad que la administración estatal puede sentir al actuar sobre un territorio concreto siendo, como es, una administración sin competencias territoriales.

Desde la perspectiva funcional, puede argumentarse que la negociación con las Comunidades Autónomas u otras administraciones plantea ventajas al Ministerio de Fomento, al configurar un frente «a favor del proyecto» al que difícilmente puede oponerse cualquier otro agente de manera eficaz. A su vez, esta situación facilita el que las distintas fases de información pública y de aprobación, donde pueden ser necesarios informes y dictámenes de esas administraciones, puedan resolverse en plazos breves y sin polémica. El hecho de que los mecanismos de participación ordinaria sean débiles dificulta, obviamente, el que puedan establecerse en esas circunstancias posturas efectivas de oposición al proyecto.

Con todo, el proceso de negociación es singular, puesto que las posiciones de las partes son francamente asimétricas: por un lado, una administración que va a realizar la totalidad de la inversión; por el otro, una administración que, sin comprometerse generalmente en la aportación de recursos, exige la ejecución de una obra, con frecuencia pidiendo características de diseño más costosas y plazos de ejecución abreviados: las expectativas de poder asistir en estas condiciones de partida a una negociación en base a razones y buenos argumentos parecen muy escasas.

La perspectiva competencial puede matizar esta impresión. Se reflejaba en un apartado anterior la fuerte tradición de una visión técnica que concibe las infraestructuras de transporte como instrumento de una ordenación del territorio, y la imposibilidad desde la administración del Estado de plantear una perspectiva o proyecto territorial. Al entrar en negociación con la administración competente en el territorio, el Estado encuentra un modo legítimo de abordar esa reflexión territorial, y además intenta contar con garantías de que la implantación inicial de la infraestructura y su operación futura no estarán afectadas negativamente por decisiones de ordenación territorial (la creación de una zona urbanizada, la ocupación de terrenos previstos para alguna infraestructura complementaria, etc). 
No puede constatarse, sin embargo, que este diálogo incluya un esfuerzo de concertación sobre los objetivos del proyecto, es decir, que intente la búsqueda de una visión compartida (aunque fuera parcial) en cuanto al modelo territorial o en cuanto a las pautas de movilidad en el futuro. Posiblemente sea éste el mayor reproche que cabe hacer a este tipo de concertación: el que no sólo no está basado en un cierto consenso previo sobre los objetivos sectoriales (movilidad) o territoriales, sino que ni tan siquiera parece realizar un debate sobre los méritos y riesgos asociados a la actuación dentro del contexto más amplio del tipo de movilidad y de desarrollo territorial que se está promoviendo desde cada administración. Actuar sobre un territorio metropolitano, por ejemplo, sin enmarcar la actuación en unos objetivos sobre reparto modal o gestión de la demanda de movilidad parece temerario. Hacerlo sobre un territorio interurbano sin explicitar la estrategia de ordenación territorial de la zona, y su compatibilidad con la infraestructura propuesta, es sin duda situarse en una posición de riesgo con respecto a la expectativa de obtención de beneficios tangibles a cambio de la inversión.

Estas reflexiones permiten inferir que las administraciones abordan esta cooperación desde una perspectiva de partida claramente «a favor del proyecto», o al menos «a favor de realizar la inversión», más que de «evaluar el proyecto», y que adoptan gustosas sus papeles respectivos: el de ejecutor eficiente de la inversión en un caso, el de guardián celoso de los derechos del territorio a recibir esa inversión, en el otro.

Desde la perspectiva de la formalización, es preciso recordar que el sector del transporte cuenta con una formalización escasa tanto de las fases de planificación como de los procesos de participación y concertación. Esto convierte los procesos iniciales de toma de decisiones en un «territorio sin mapa» en el que se trabaja con documentos técnicos ad hoc y donde el acceso a la información, y la transparencia en general sobre las decisiones adoptadas, resultan escasas.

Esta situación supone un obstáculo infranqueable para la puesta en marcha de los instrumentos que en otros países están contribuyendo a mejorar los resultados de la planificación y su legitimidad y, en definitiva, a consolidar sistemas de toma de decisiones mejor integrados en la perspectiva de desarrollo sostenible. Este es el caso por ejemplo de la Evaluación Ambiental Estratégica, que tras aplicarse al caso del PEIT no ha podido aplicarse a documentos de planificación de mayor detalle simplemente porque esos documentos no han llegado formalmente a someterse al proceso de evaluación previsto en la Directiva 2001/42/CE y en la Ley 9/2006, lo que no ha impedido continuar la tramitación desde el nivel de estudio informativo (o equivalente) de cada actuación, produciendo, de hecho, una situación similar a la existente con anterioridad a la entrada en vigor de la Directiva.

Por otra parte, el artículo 3.1.b) de la Ley 9/2006 establece que el ámbito de aplicación de la EAE está limitado a aquellos planes y programas cuya «elaboración y aprobación venga exigida por una disposición legal o reglamentaria o por acuerdo del Consejo de Ministros o del Consejo de Gobierno de una comunidad autónoma». Paradójicamente, los planes de transporte no viene exigidos por las disposiciones 
legales, y la tentación para el gestor de evita el molesto trámite de la EAE para sus planes es probablemente demasiado fuerte.

Puede concluirse que, sin un esfuerzo efectivo de formalización de la fase de planificación, incluyendo el mandato legal de la elaboración de planes de transporte generales y sectoriales, el fortalecimiento de los mecanismos de participación y una mejor vertebración del proceso de concertación con las Comunidades Autónomas, será prácticamente inviable consolidar un marco que supere el poco eficiente statu quo existente. Una tarea urgente, puesto que en el entorno europeo sí se están produciendo importantes reformas, justificadas desde la necesidad de adaptar la política sectorial al paradigma de desarrollo sostenible y a un marco de gobernanza en las políticas públicas más transparente y mejor vinculado a la eficiencia en la consecución de objetivos explícitos.

El mantenimiento del statu quo facilita, además, la adopción de decisiones, posiblemente justificadas desde una perspectiva coyuntural, que restan viabilidad al proyecto de «cambio de rumbo» del sistema de transporte planteado en la planificación estratégica. Es el caso, por ejemplo, de algunas decisiones en infraestructuras sobre la red ferroviaria de alta velocidad (por ejemplo, algunos tramos que se diseñan sin posibilidad para su uso de mercancías haciendo inviable el esquema de tráfico mixto del plan) o en la normativa (el bloqueo de la Directiva sobre la Euroviñeta por parte de España a pesar de estarse planteando en el Plan la necesidad de establecer medidas para favorecer el cambio modal hacia los modos más sostenibles).

En definitiva, puede concluirse que es fácil constatar una enorme debilidad de las etapas estratégicas de la planificación al menos hasta la fase en la que se estudia cada actuación individualizada (estudio informativo o equivalente), y que la falta de una formalización adecuada a través de la normativa contribuye de forma determinante a esta situación.

\section{El papel de la evaluación económica: perspectivas}

La evaluación económica de proyectos de transporte ha estado tradicionalmente limitada en España a la comparación de alternativas, y sus resultados se integran con los procedentes de otros análisis (en particular los ligados a la evaluación ambiental), formando así parte de un conjunto de información más amplio que se pone a disposición de los diversos agentes dentro del proceso de información pública, y de los responsables de la toma de decisiones.

Sin embargo, la evaluación económica no se emplea habitualmente para descartar dentro del sistema de planificación los malos proyectos. Esto sí se hace en algunos países: por ejemplo, en Alemania, durante la fase de preparación del plan federal de transporte se utilizan tres tipos de evaluación: la socio-económica, la ambiental y la territorial; estas evaluaciones son muy someras, pero sirven para descartar proyectos que se consideran claramente inadecuados, por ejemplo por presentar un tasa interna de retorno claramente insuficiente, o por afectar fuertemente 
zonas ambientales con una protección elevada (como la red Natura 2000). En los Países Bajos desde finales de los años 90 se ha estado realizando una evaluación coste-beneficio en la fase de planificación de los grandes proyectos de transporte con arreglo a una metodología claramente definida (Annema, 2007); se aplica a proyectos de elevado coste y, a diferencia del caso alemán, se trata de una evaluación socioeconómica que integra tanto las externalidades como los efectos indirectos sobre el desarrollo regional; los resultados de esta iniciativa, sin embargo, no parecen haber estado a la altura de las expectativas. El Reino Unido es, posiblemente, el país europeo en el que la evaluación socioeconómica está más consolidada y donde tiene una mayor influencia en la toma de decisiones (ITF, 2008).

Francia, donde la evaluación socioeconómica también ha alcanzado un desarrollo importante, ha evolucionado progresivamente hacia una consideración cada vez mayor de los objetivos estratégicos ligados al desarrollo sostenible y lo que puede denominarse, en definitiva, como integración de esos objetivos en la política sectorial de transporte. En consecuencia, la toma de decisiones en las etapas iniciales de planificación se ha vinculado, durante la primera parte de la década del 2000, a la concertación sobre el modelo territorial, a través del Comité Interministériel d'Aménagement et de Développement du Territoire (CIADT) y, a partir del lanzamiento en 2007 del proceso «Grenelle de l'Environnement», a la aplicación de los principios acordados en el proceso y, después, de las dos leyes en las que se han plasmado. El resultado es que la evaluación socioeconómica se retrasa hacia las fases posteriores dentro de la definición del proyecto, y que las decisiones en la etapa de planificación se centran en consideraciones de tipo territorial y, sobre todo, de compatibilidad con el modelo de «movilidad sostenible» definido por las leyes Grenelle. Puede encontrarse en el avance de documento de planificación estratégica del transporte recientemente publicado (MEEDDM, 2010), algunos paralelismos formales en la metodología de planificación con respecto al PEIT.

¿Es posible, y en su caso conveniente, el utilizar la evaluación económica en fases anteriores de la planificación? Es evidente que existe en nuestro país, y también entre los profesionales en otros países, una cierta prevención hacia el uso de esta herramienta en esa fase. Sin abundar en las limitaciones metodológicas, y en la incertidumbre sobre la mayoría de los datos que se utilizan como base para los cálculos, sobradamente debatidos y conocidos (véase por ejemplo Metz, 2008; Welch, 1997), pueden señalarse dos consideraciones: en primer lugar, la dificultad para vincular los resultados del ACB a los objetivos de la planificación; en segundo lugar, la evidencia en algunos casos de cómo el ACB puede utilizarse para justificar actuaciones que no parecen consistentes.

La primera cuestión hace referencia a las grandes preocupaciones actuales sobre el sistema de transporte y las estrategias de actuación que se están poniendo en marcha. En un contexto europeo de impulsión de modos de transporte con menor impacto sobre el medio ambiente (transporte público urbano, transporte de mercancías multimodal apoyado en el ferrocarril, transporte de personas multimodal, apoyado 
en la alta velocidad ferroviaria) no parece que estas prioridades puedan plasmarse adecuadamente en una metodología en la que el 70-90 por 100 de los beneficios están ligados a los ahorros de tiempo, es decir, al aumento de la velocidad, donde la ventaja mayor es para el transporte aéreo o para el transporte «puerta a puerta» ofrecido por la carretera. Quizá esto pudiera ser una indicación bien de que los objetivos de la política de transporte en estos momentos son contrarios a la lógica económica, bien de que la lógica económica está demasiado alejada de algunos de los desafíos principales de nuestro tiempo, pero abordar esta cuestión excede con mucho el ámbito de este trabajo. El hecho es que en términos generales, los proyectos del tipo «business as usual» presentan frecuentemente resultados más favorables que los proyectos que suponen una cierta ruptura con las políticas de intervención tradicionales, y además cuentan a su favor con una cuantificación de las variables ya consolidada y generalmente aceptada, cosa que no es el caso en el segundo tipo de proyectos, que intentan provocar cambios en las pautas de movilidad, más inciertos y difíciles de prever.

Esta reflexión sugiere que, de hecho, el ACB puede ofrecer información útil para comparar entre alternativas o variantes dentro de un proyecto en una situación bien acotada o definida, pero no para hacerlo en el contexto abierto de las fases iniciales de planificación. Esto conduce a una segunda cuestión: la evidencia de que la aplicación del ACB puede justificar soluciones poco consistentes, especialmente cuando se contemplan actuaciones novedosas o se trabaja en un entorno particularmente complejo; finalmente, parece que el ACB puede fácilmente ponerse al servicio de la justificación de una actuación previamente decidida. Dos ejemplos relativamente recientes pueden ilustrar estos argumentos: se trata del ACB realizado para la remodelación de la autopista de circunvalación M-30 en Madrid (el estudio se realiza en 2004, coincidiendo con el comienzo de las obras, que se finalizan en 2007) y del ACB realizado en 2006 dentro del estudio informativo para la construcción de una vía reservada a autobuses en la A-42, una de las autovías radiales de entrada en Madrid. Los dos casos resultan interesantes, pues presentan resultados favorables para la adopción de un tipo de solución que supone la ampliación de la capacidad del viario existente (lo que se traduce en las consiguientes ganancias de tiempo para los usuarios): un tipo de política que ha sido descartado desde hace décadas en el entorno europeo porque consolida la utilización del vehículo privado $\mathrm{y}$, a largo plazo, la dispersión urbana.

El primer caso (UPM, 2004) no estudia alternativas de actuación. Al tratarse de un estudio realizado después de que la decisión haya sido adoptada, su propósito es el de ofrecer argumentos sobre la racionalidad de un proyecto ya en ejecución. La comparación se realiza entre la situación con proyecto y sin proyecto suponiendo que la puesta en servicio se realiza en 2007 y estableciendo un período para el análisis de treinta años, es decir, hasta 2037.

El estudio muestra numerosos ejemplos de la amplia flexibilidad que ofrece el ACB al profesional para conseguir los resultados deseados empleando las variables adecuadas a su propósito: así, se utiliza una tasa de descuento sorprendentemente 
baja, del 2,44 por 100 anual; se mantiene el reparto modal inicial a lo largo de los 30 años de evaluación, a pesar del considerable deterioro que se produce en las condiciones de circulación; se establece un valor del tiempo elevado, de 9 ? por persona y hora ${ }^{9}$, y se considera un crecimiento notable de la movilidad en vehículo privado año a año (del 1,75 por 100 anual para el período 2007-2012 y del 1,10 por 100 anual para el período 2012-2037); no se consideran las reducciones de velocidad que el nuevo proyecto implica para buena parte de la vía (los tramos en túnel pasan a tener una velocidad máxima autorizada de $70 \mathrm{~km} / \mathrm{h}$ frente a los $90 \mathrm{~km} / \mathrm{h}$ con que contaban esos tramos cuando estaban en superficie); no se revisan los datos iniciales de coste del proyecto (3.663 millones de euros), a pesar de la evidencia ya en aquel momento de que los costes serían muy superiores ${ }^{10}$. Finalmente, las hipótesis de cálculo resultan contradictorias con los objetivos habituales de una estrategia de movilidad urbana en Europa: gestionar la demanda (intentando reducir el crecimiento del número de viajes) y disminuir la utilización del vehículo privado.

El resultado es una tasa interna de retorno del 5,24 por $100 \mathrm{y}$ un valor actual neto de 1.748 millones de euros. Beneficios y costes no se reparten uniformemente en toda la actuación, sino que la mayor parte de los beneficios proceden de las reformas en el tramo Este, que son las menos costosas: suponen un 12 por 100 del presupuesto, pero aportan el 86 por 100 del VAN, pero esto no lleva a reflexión alguna sobre la evaluación por separado de tramos que, funcionalmente, podían considerarse como proyectos separados.

El segundo ejemplo corresponde a la fase de estudio informativo de la reforma de $27 \mathrm{~km}$ en la autovía 42 en Madrid, entre un punto próximo a la autovía de circunvalación M-30 (la plaza de Fernández Ladreda) y el límite de la provincia de Madrid. De hecho, este estudio informativo retomaba un proyecto de mediados de los años 90, abandonado después, para la construcción de una vía central de dos carriles en la autovía, uno para cada sentido, para uso exclusivo de autobuses ${ }^{11}$.

El proyecto introduce dos novedades que nada tienen que ver con el fomento del transporte público y que, de hecho, no hacen sino aumentar el atractivo del vehículo privado: añade un tercer carril por sentido de circulación y establece vias de servicio laterales de dos carriles en todo el tramo. El resultado del análisis ACB es que la mejor opción es realizar esta ampliación de capacidad para el vehículo privado (es decir, el tercer carril más las vías de servicio) y no ejecutar la calzada central para

9 El valor del tiempo es otro elemento de debate. Valores del tiempo elevados, como este, no parecen recoger con fidelidad la realidad de las decisiones de los usuarios: por ejemplo, el nivel real de utilización de las autopistas de peaje sugiere que los valores reales del tiempo son considerablemente inferiores.

10 Información del Ayuntamiento señala que el coste final se estima en unos 3.330 millones de euros, pero hay que tener en cuenta que hasta el momento no se han realizado la mayor parte de las obras previstas en el tramo norte, que tenían un presupuesto de más de 1.000 millones de euros. Otras informaciones hablan de un coste final de 4.800 millones.

11 Ministerio de Fomento; Demarcación de Carreteras del Estado de Madrid. Estudio Informativo: «Optimización funcional de la Carretera A-42 entre la Plaza Fernández Ladreda y Torrejón de la Calzada», y de su documento complementario: «Estudio Complementario 
autobuses. Esta conclusión se corrige después en el análisis multicriterio, incorporando también, «por consideraciones ambientales», la calzada central.

El ACB muestra que la principal aportación al VAN no procede de los usuarios del transporte público, sino del resto de usuarios. El planteamiento del ACB no ha tenido en cuenta la lógica inicial con la que se plantea esta actuación desde la planificación: crear una ventaja diferencial al transporte público respecto del resto del tráfico, no incrementar ciegamente la capacidad de la vía para todos los vehículos.

Los dos ejemplos muestran además otro tema que ha sido polémico durante muchos años. Aunque no se dispone en ninguno de los estudios de una distribución de los ahorros de tiempo, una comparación entre los valores agregados de tiempo ahorrado y el número de usuarios sugiere que una gran parte del ahorro total se produce al sumar pequeños ahorros de tiempo (inferiores a 3-5 minutos) de un número muy elevado de usuarios. En el ámbito académico y profesional no hay consenso sobre lo correcto de este planteamiento: por una parte, se señala que estos pequeños ahorros de tiempo difícilmente pueden ser aprovechados e incluso percibidos por los usuarios; por la otra, se aportan dos argumentos para defender el tenerlos en cuenta: el de la distribución (para algunos usuarios estas ganancias tendrán ciertamente poco o ningún valor, pero para otros suponen una diferencia considerable, al poder sumarse a otras ganancias latentes pero hasta ese momento insuficientes para superar un cierto umbral de tiempo que le permite realizar cambios con un impacto económico importante) y el de la descomposición (las ganancias económicas ligadas a los ahorros de tiempo se producen de manera incremental, sumando las pequeñas ganancias obtenidas de múltiples actuaciones, por tanto la única manera de realizar el cálculo es tener en cuenta cada pequeña ganancia en cada proyecto, porque no podemos pretender identificar dentro de los usuarios entre los que con un proyecto determinado consiguen realmente poner en valor el ahorro y los que no) (véase Welch, 1997 para una discusión detallada de estos argumentos). En la práctica, algunos países como Alemania, introducen un umbral mínimo de tiempo y otros, como el Reino Unido, no lo hacen. En los dos casos aquí presentados se trata, sin embargo, de una situación extrema, donde posiblemente la mayor parte del ahorro de tiempo total corresponde a este tipo de pequeños valores.

Una segunda consideración es que la evolución pronosticada para las condiciones de tráfico, tanto en el escenario con proyecto como sin proyecto, no parece consistente con la experiencia. En los estudios se produce en el tiempo un progresivo deterioro de las condiciones de tráfico en todas las alternativas, pero las alternativas con menor capacidad se deterioran de manera más acusada, de manera que el diferencial en las velocidades medias entre la situación con proyecto y sin proyecto aumenta con el paso del tiempo. Sin embargo, la experiencia muestra que el tráfico urbano no se comporta de esta manera, y que cuando se alcanza un cierto nivel de saturación se producen efectos de distribución dentro de la red (asociados a la ejecución de otros proyectos), de cambio modal, o incluso de cambios en el origen y destino (buscando puntos de residencia y actividad con mayor accesibilidad). De particular interés es el hecho de que el llamado «presupuesto de viaje», es decir, el 
tiempo dedicado diariamente al transporte, parece ser muy constante (una media de unos 90 minutos en el caso del Reino Unido, Metz, 2008): los ahorros de tiempo se transforman, a medio plazo, en trayectos más largos o en viajes adicionales. Este argumento justificaría, en proyectos en situaciones de congestión, típicos del medio urbano, el considerar que, a partir de unas ciertas condiciones de tiempo de viaje, una parte de los usuarios desarrolla estrategias alternativas (cambio de horario, cambio de modo, cambio de origen o destino), de manera que la situación del tráfico permanece estacionaria. Esta es la experiencia práctica de la poco eficiente, pero sin embargo generalizada, gestión de la demanda por la congestión, presente en muchas ciudades europeas, que explica que unas condiciones de tráfico deterioradas no sigan degradándose y que, cuando se realiza alguna mejora, aparezca nuevo tráfico que rápidamente anula la ventaja conseguida.

Desde esta perspectiva, no resultaría razonable el continuar añadiendo los ahorros de tiempo futuros a partir del año en el que la velocidad media de desplazamiento bajara, en la situación «con proyecto» $\mathrm{y}$ «sin proyecto» por debajo de un cierto valor. Esta situación sería, además, más consistente con el rango de validez de los modelos de tráfico, que resultan poco adecuados para reproducir situaciones de congestión.

Estos dos ejemplos ilustran alguno de los interrogantes que desde la planificación y más aún desde la toma de decisiones, se plantean sobre el ACB. Reconociendo su capacidad para sintetizar una información compleja, se considera que es útil en la comparación de alternativas de actuación de una cierta similitud, una vez que el grado de definición del proyecto está relativamente definido y el rango de alternativas está acotado, pero que difícilmente puede pretender recoger la complejidad de unos objetivos de política de transporte cada vez más integrados en una perspectiva multisectorial, sea desde el paradigma del «desarrollo sostenible» o desde otros. En definitiva, la práctica del ACB pone de manifiesto que puede favorecer alternativas que no son razonables desde una perspectiva de movilidad sostenible.

\section{Conclusiones}

En este contexto, puede argumentarse que la utilización del ACB parece servir como un obstáculo al despliegue de políticas más radicales en España para la reforma del sistema de transporte desde una perspectiva de sostenibilidad, y que difícilmente puede considerarse como una herramienta convincente, dentro del proceso de toma de decisiones, para descartar un mal proyecto. La mayor parte de las decisiones sobre infraestructuras de transporte que pueden considerarse como no eficientes, y donde el ACB podría aportar información, de hecho pueden detectarse como tales desde un análisis multicriterio, o desde estudios ad hoc. El problema no es que esas ineficiencias no hayan sido detectadas y analizadas desde los servicios técnicos y desde las instancias de toma de decisión, sino que el marco de definición 
y de ejecución de políticas públicas favorece que algunas propuestas de inversión se carguen de valores simbólicos que conducen a su ejecución a pesar de su escasa rentabilidad, cuando no de su evidente contradicción con los objetivos territoriales y de transporte. La proliferación de esos proyectos en España en los últimos años debe servir como toque de atención sobre la necesaria reforma del sistema de toma de decisiones, para que pueda contrarrestar la fuerza de esos «valores simbólicos».

En cualquier caso, el ACB es per se extremadamente manipulable desde el ámbito técnico. Su empleo debería ir vinculado, como en los casos del Reino Unido, Francia, Alemania y Países Bajos, con el desarrollo de unas directrices metodológicas mucho más desarrolladas que de las que se dispone por el momento. La experiencia de Países Bajos en estos últimos diez años muestra que esto exige un considerable esfuerzo teórico inicial, y un trabajo aún mayor de seguimiento y revisión en años posteriores. Este esfuerzo puede resultar útil, pero ni es imprescindible ni es prioritario respecto al de la reforma del sistema de toma de decisiones. En ese ámbito, la formalización del proceso, la utilización reforzada de los mecanismos que proporciona la evaluación ambiental, y la puesta en marcha de procesos de diálogo más trasparentes puede tener un efecto más relevante. Demorar esta reforma, y vincular la búsqueda de eficiencia en las tomas de decisión «estratégicas» al ACB sería un error, dando una preeminencia a la racionalidad económica sobre otros argumentos importantes, y haciéndolo desde una «ilusión» de objetividad que no se corresponde con la realidad de la práctica ACB.

\section{Referencias bibliográficas}

[1] ANEGÓN, C.; APARICIO, Á. y SÁNCHEZ VICENTE, A. (2009): El transporte en España; informe basado en el sistema de indicadores de seguimiento del transporte y su impacto ambiental (SISTIA, 2009). Madrid: CEDEX.

[2] ANNEMA, J. A., y KOOPMANS, C. et al. (2007): «Evaluating Transport Infrastructure Investments: The Dutch Experience with a Standardized Approach.» Transport Reviews 27(2): 125-150.

[3] COMITÉ DE DESARROLlO TERRITORIAL (CDT) (1999): Estrategia Territorial Europea: Hacia un desarrollo equilibrado y sostenible del territorio de la UE. Luxemburgo: Comunidades Europeas.

[4] DAMART, S. N. y ROY, B. (2007): «Limitations of Cost Benefit Analysis to support public debate: the case of public Transportation Decision-making in France».

[5] EUROPEAN COMMISSION; expert group 1 «methodology ofr TEN-T planning» (2010). Proposal on TEN-T Network Planning. http://ec.europa.eu/transport/infrastructure/tent_policy_review/ expert_groups/doc/final_report_ten-t_planning_methodology_eg1.pdf.

[6] EUROPEAN CONFERENCE OF MINISTERS OF TRANSPORT (2004). Assessment \& Decision Making for Sustainable Transport. Paris: ECMT.

[7] EUROPEAN ENVIRONMENT AGENCY (EEA) (2009): Transport at a crossroads: 
TERM 2008: indicators tracking transport and environment in the European Union. Copenhagen, EEA.

[8] FORESTER, J. (2008). Editorial. Planning Theory \& Practice, vol. 9, nº 3, pp. 299-304.

[9] GONZALO ORDEN, H.; ROJO ARCE, M. y DELL'OLIO, L. (2008):«Las nuevas infraestructuras viarias previstas en Castilla y León: efectos socioeconómicos y territoriales». Estudios de Construcción y Transportes/n.108 (en.-jun.2008); p.81-101.

[10] GUTIÉRREZ PUEBLA, J. et al. (2006): «Análisis de los efectos de las infraestructuras de transporte sobre la accesibilidad y la cohesión regional». Estudios de Construcción y Transportes/n.105 (jul.-dic.2006); pp. 215-240.

[11] HEALEY, P. (2006): Collaborative Planning: Shaping Places in Fragmented Societies. New York, Palgrave MacMillan.

[12] HYMEL, K. M., y SMALL, K. A. et al., (2010): «Induced Demand and Rebound Effects in Road Transport». Transportation Research Part B In press (August 2010).

[13] INNES, J. E. B., y DAVID, E. (2004): «Reframing public participation: Strategies for the 21st century». Planning Theory \& Practice, vol. 5, n. ${ }^{\circ}$ 4, pp. 419-436.

[14] JONG, M. D., y GEERLINGS, H. et al. (2007): «In search of the ideal institutional structure for decision-making on transport infrastructure : a conjoint analysis of expert opinions on hybrid forms of German, Danish and Dutch decision-making». Transport project evaluation : extending the social cost-benefit approach.: 197-216.

[15] LÓPEZ SUÁREZ, E. (2009): «Análisis de impactos territoriales del Plan Estratégico de Infraestructuras y Transporte 2005-2020: Cohesión regional y efectos desbordamiento». Información Comercial Española, n. ${ }^{\circ} 847$ (marzo-abr. 2009), pp.159-172.

[16] METZ, D. (2008): «The Myth of Travel Time Saving». Transport Reviews, 28: pp. 321336.

[17] MINISTÈRE DE L'ÉCOLOGIE, DE L'ÉNERGIE, DU DÉVELOPPEMENT DURABLE ET DE LA MER (2010): Schéma nacional des infrastructures de transport soumis à concertation. Paris: MEEDDM.

[18] MINISTERIO DE FOMENTO (2007). Primer balance del PEIT.

[19] MINISTERIO DE FOMENTO (2005). Plan Estratégico de Infraestructuras y Transporte (PEIT) 2005-2020. Madrid: Ministerio de Fomento.

[20] MINISTERIO DE FOMENTO (2004) Informe de sostenibilidad ambiental del PEIT.

[21] MINISTERIO DE OBRAS PÚBLICAS, TRANSPORTES Y MEDIO AMBIENTE (MOPTMA) (1994). Plan Director de Infraestructuras 1993-2007. Madrid: MOPTMA.

[22] ODECK, J. (2010). «What Determines Decision-Makers' Preferences for Road Investments? Evidence from the Norwegian Road Sector». Transport Reviews 30(4): pp. $473-$ 494.

[23] STECHER, B. M. (2010). Toward a culture of consequences : performance-based accountability systems for public services, RAND Corporation.

[24] TRANSPORT RESEARCH CENTRE, International Transport Forum (ITF), et al. (2008). The wider economic benefits of transport : macro-, meso- and micro-economic transport planning and investment tools. Paris, OECD/ITF.

[25] TUDELA, A. y AKIKI, N. et al. (2006). «Comparing the output of cost benefit and multi-criteria analysis». Transportation Research Part A 40(5):pp. 414-423.

[26] UPM (2004) Programa de mejoras del anillo de circunvalación M-30 de Madrid: evaluación socio-económica y ambiental.

[27] VAN DER KRABBEN, E. y Martens, K. et al. (2008): «How to Define the Optimal 
Level of Public-sector Infrastructure Development? A Conceptual Model for Decisionmaking in Infrastructure Projects». Planning Practice and Research 23(3): pp. 363381.

[28] WELCH, M. y WILLIAMS, H. (1997): «The Sensitivity of transport Investment Benefits to the Evaluation of Small Travel-Time Savings». Journal of Transport Economics and Policy 31(3): 24. 